\title{
A Novel Technique to Improve Gain In Transparent UWB Antennas
}

\author{
T. Peter ${ }^{\# 1}$, T.I. Yuk ${ }^{* 2}$, R. Nilavalan ${ }^{\# 3}$, S.W. Cheung ${ }^{* 4}$ \\ ${ }^{\#}$ Dept of Electronics and Computer Engineering, Brunel University, West London \\ *Department of Electrical and Electronic Engineering, University of Hong Kong \\ ${ }^{1}$ Thomas.Peter@brunel.ac.uk \\ ${ }^{4}$ swcheung@eee.hku.hk
}

\begin{abstract}
A novel technique to improve the performance of AgHT-8 transparent polymer antennas is proposed in this paper. A spit-ring resonator is introduced on the radiating patch to enhance gain. The resonator basically concentrates the radiating energy to the central area of the patch thus improving gain. The designed antenna demonstrates good gain while maintaining the original transparency of the material. Such an antenna inscribed on the commercially available AgHT-8 sun shielding film material makes it a viable option for wireless applications like in-house base stations and applications requiring fast data rate transfers which can be mounted on windows and glass panels.
\end{abstract}

\section{Index Terms - Transparent, UWB, Arrays, Gain, Glass}

\section{INTRODUCTION}

$\mathrm{T}$ RANSPARENT antennas have in recent tin having great potentials for wireless applic growing interest for integrating such panels to harvest energy whilst communication facilities [2]. Hoy ane or notable problems in such type of tray arent an nas acially, those made from AgHT-8, is low gain. Im pving gain on these antennas which are mado m transpar materials that are lossy in nature is ifficul reason could be alluded to the 3-layer conductive k. A possible rial composition of AgHT-8; the primary conducting layd, that is the central silver layer is overlayed with tin oxide which makes it lossy[3]. In previous works, [3] efficiency was shown to be improved by $20 \%$ using a novel soldering technique. However, this improvement is not enough to attract commercial interest to the use of AgHT-8 as an antenna material. Gain improvement for better transmission and reception should additionally be explored to make the transparent antennas a good commercial option.

In this paper, a technique to improve the gain of transparent UWB antennas is introduced. Implementing the same technique in [3] and using resonators to expose and concentrate the radiating energy around the central area of the patch, the gain is shown to have improved. The split ring resonator basically introduces capacitance. At low frequencies this 'split ring capacitor' acts as an open circuit, as a result hardly any increase in gain is noticed. At higher frequencies, opposition to current flow becomes less causing the gain to increase. Increasing the size of the split ring resonator increases the gain. However, in this paper, only one size is fabric to demonstrate the concept of gain enhancemen through this technique. A comparative reference design with no resonator is also fabricated and masured to ppare the effectiveness of the split ring res ator technique. 1 shows the layout of the reference enna without a reso ator whilst Fig. 2 with a split ring Conat The antenna in this paper is designed as a single la) Aenna, that is, it is not additionally mounted on to a PET strate as in [3] or situated on a glass substrate as in [4] or a Per $x$ substrate and superstrate as in [5] so that it can aribed easily on to the AgHT-8 film and be spread onto glass panel or window without any increase in the profile of the film. The glass and Perspex techniques to enhance gain may not be suitable for glass window applications. What is needed is a thin profile planar antenna that has improved gain achieved by introducing geometrical variations into the shape and surface of the antenna and not through external optical amplification that may be achieved with glass or other materials with optical properties. This type of antenna can then be used a standalone antenna for wireless applications.

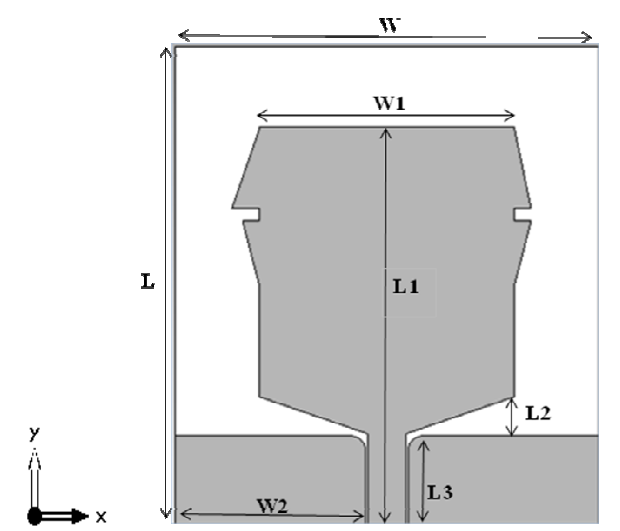

Fig. 1 Layout of reference antenna

TABLE I: Dimensions of the antenna structure in Fig.1

\begin{tabular}{|c|c|c|c|c|c|c|c|}
\hline Parameters & L & $\mathbf{L}_{1}$ & $\mathbf{L}_{2}$ & $\mathbf{L}_{3}$ & $\mathbf{W}$ & $\mathbf{W}_{1}$ & $\mathbf{W}_{2}$ \\
\hline Size (mm) & 23.4 & $\mathbf{1 9 . 5}$ & $\mathbf{1 . 8}$ & $\mathbf{4 . 4}$ & $\mathbf{2 0}$ & $\mathbf{1 2}$ & $\mathbf{9}$ \\
\hline
\end{tabular}




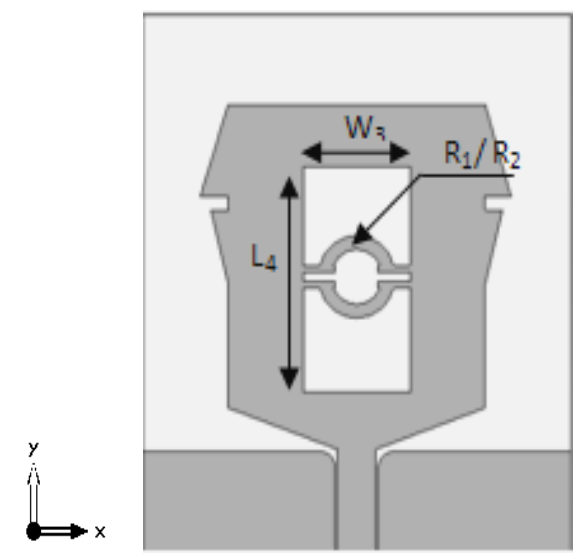

(a)
TABLE II: Dimensions of the antenna structure in Fig.2

\begin{tabular}{|c|l|l|l|l|}
\hline Parameters & $\mathbf{L}_{4}$ & $\mathbf{W}_{3}$ & $\mathbf{R}_{1}$ & $\mathbf{R}_{2}$ \\
\hline Size (mm) & 9.8 & 5 & 1.8 & 1.2 \\
\hline
\end{tabular}

Fig. 2 (a) Layout of antenna incorporating a split ring resonator

\section{AGHT-8}

The film is a polyethylene terephthalate (PET) with a three-layered transparent conductive coating of silver sandwiched between two layers of tin oxide. The film has a surface resistance of $8 \Omega$-Sq which is equivalent to a conductivity $(\sigma)$ of $1.25 \times 10^{5} \mathrm{~S} / \mathrm{m}$ [1], and a total thickness of $0.175 \mathrm{~mm}$. However, the coating thickness is around $0.052 \mathrm{p}$ or $52 \mu \mathrm{m}$. The DC conductivity of the film was measured $50000 \mathrm{~S} / \mathrm{m}$ using the four-probe technique. The PET has dielectric of 3.24. Using the formula in Equation 1 [6], the skil depth of the AgHT- 8 was estimated at $45 \mu \mathrm{m}$ at 1 $6.85 \mathrm{GHz}$ and $14 \mu \mathrm{m}$ at $10.6 \mathrm{GHz}$. The thick more than one skin depth from $1 \mathrm{GHz}$ to higher frequencies as can be seen in the desce. values for increasing frequencies. coating is around $52 \mu \mathrm{m}$ in thickn depth is negligible.

$$
\delta=\sqrt{\frac{2 \rho}{(2 \pi f)\left(\mu_{0} \mu_{r}\right)}} \approx 503 \sqrt{\frac{\gamma}{\mu_{r} f}}
$$

\begin{abstract}
III. ANTENNA DESIGN
The tw ntennas were designed with a coplanar wavegy $(\mathrm{CPW})$ fo and fabricated using the AgHT-8 film. The diator, $\mathrm{CPW}$ fo and grounds are designed on the active ating portion. The antenna is designed as a red gul patch with bevels at the bottom portion for imped matching. Flaps are introduced on opposite edges for the angular patch for impedance matching as well as dwidth hancement. The $50 \Omega \mathrm{CPW}$ line is $1.8 \mathrm{~mm}$ in al a strip gap of $0.1 \mathrm{~mm}$ on both sides of the central feed ne. The total dimensions of the antenna including the substrate are $20 \times 20 \times 0.175 \mathrm{~mm}$. The antenna is compact and optically ansparent. The antenna with the above dimensions and parameter is designed as the reference antenna while the other antenna is designed with a cavity in the central section of the radiator and incorporated with a split ring resonator.
\end{abstract}

The antenna with the split ring resonator has a split ring resonator within a cavity in the patch. The split ring resonator is joined on both sides to the edges of the cavity as seen in Fig.2. The split ring has an external radius of $1.8 \mathrm{~mm}$ and an internal radius of $1.2 \mathrm{~mm}$. The dimensions of the ring can be increased for more gain but the above dimensions are adhered to as a proof of concept. The co-axial cables were connected to the feed points using the same soldering techniques as described in [3].

\section{MEASUREMENT AND SimUlation RESUltS}

The antennas were designed and simulated on CST Microwave Studio. The antennas performances were verified by fabricating them and measuring the return loss. The fabricated prototypes are shown in Fig. 3. 


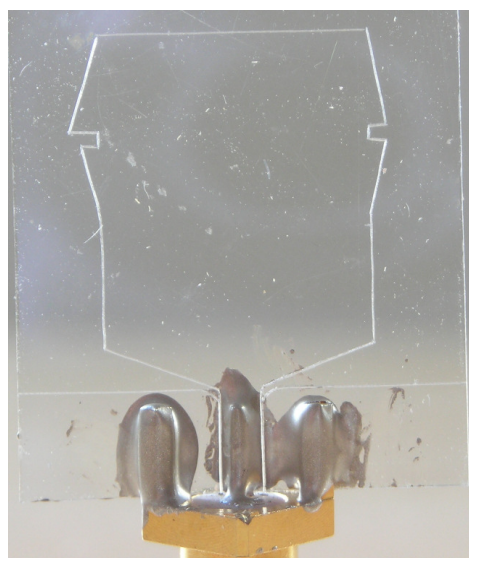

(a)

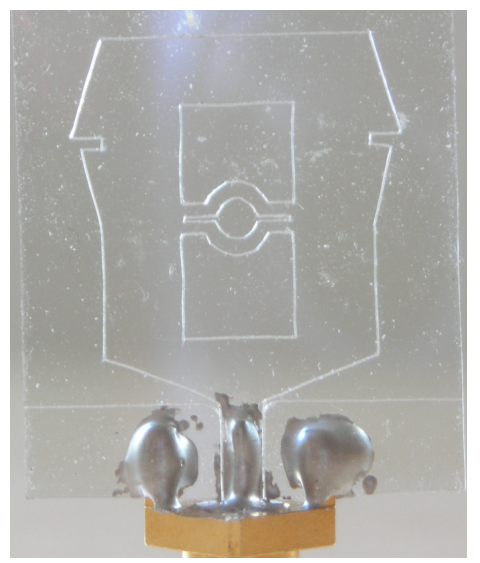

(b)

\section{Fig.3 Prototype of antennas a) Reference antenna b) Split ring resonator antenna}

The measured return losses of the two fabricated antenna prototypes are comparatively shown with the simulated results of the designed antennas with a $50 \Omega$ port in Fig. 4 . The return loss patterns of the two antennas were comparatively wide in bandwidth.

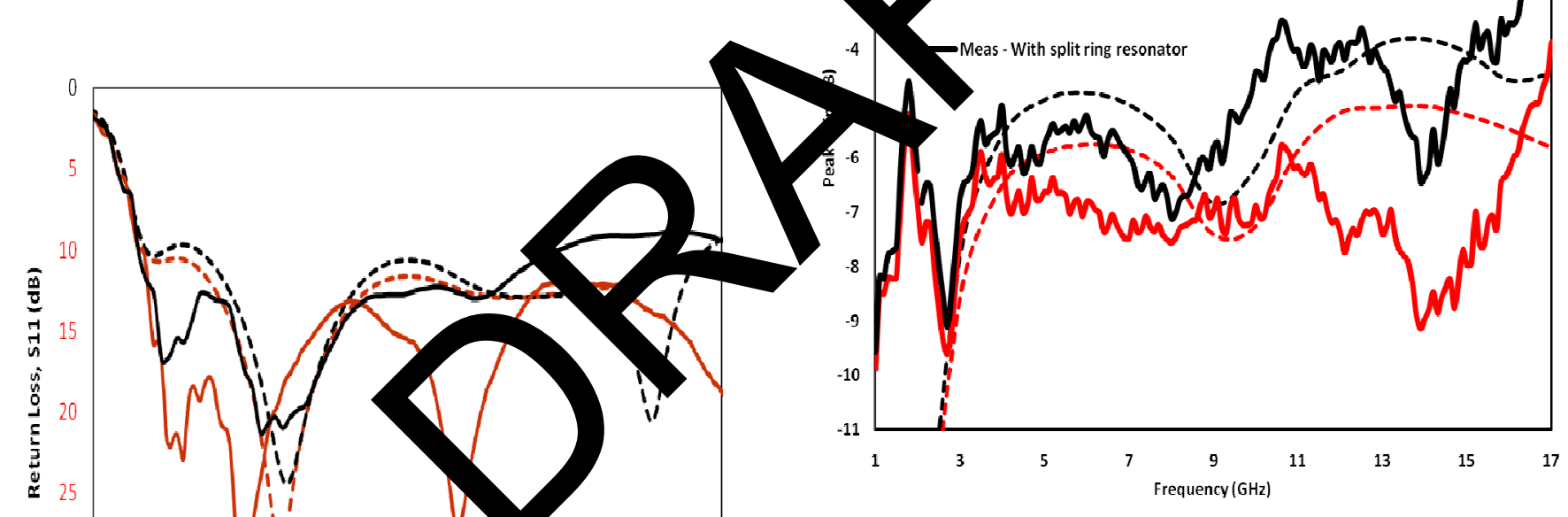

Fig. 5 Peak gains of the two prototype antennas

The gain at the lower frequencies for the split ring resonator antenna and the reference antenna were the same and showed no increase in gain. This is because at low frequencies, the 'capacitor' acts as an open circuit. The gain is shown to increase across the high frequencies of the split ring resonator. A noticeable $1.5 \mathrm{~dB}$ increase in peak gain can be seen from $4 \mathrm{GHz}$ to $9 \mathrm{GHz}$ and a $3 \mathrm{~dB}$ increase above $9 \mathrm{GHz}$ over that of the reference antenna, clearly indicating, that the split ring resonator implementation helps to enhance the gain.

Gain and radiation pattern measurements of the two fabricated prototypes were made on Satimo's antenna measurement equipment, StarLab. The measured peak gain of the two prototype antennas are as shown in Fig. (5).

The measured radiation patterns at $3 \mathrm{GHz}$ and $7 \mathrm{GHz}$ in the vertical, Y-Z plane and horizontal, X-Y plane are as shown in Fig. (6) and (7) respectively. 

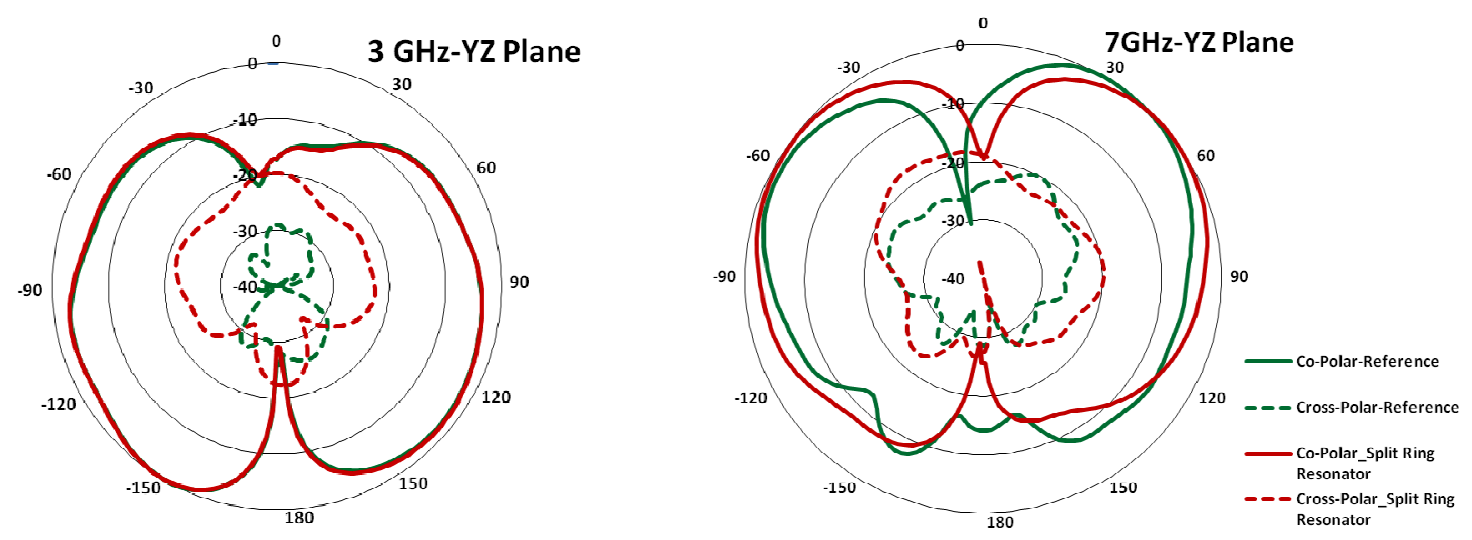

Fig. 6 Measured radiation patterns of the two antennas in the Y-Z planes at $3 \mathrm{GHz}, 7 \mathrm{GHz}$.
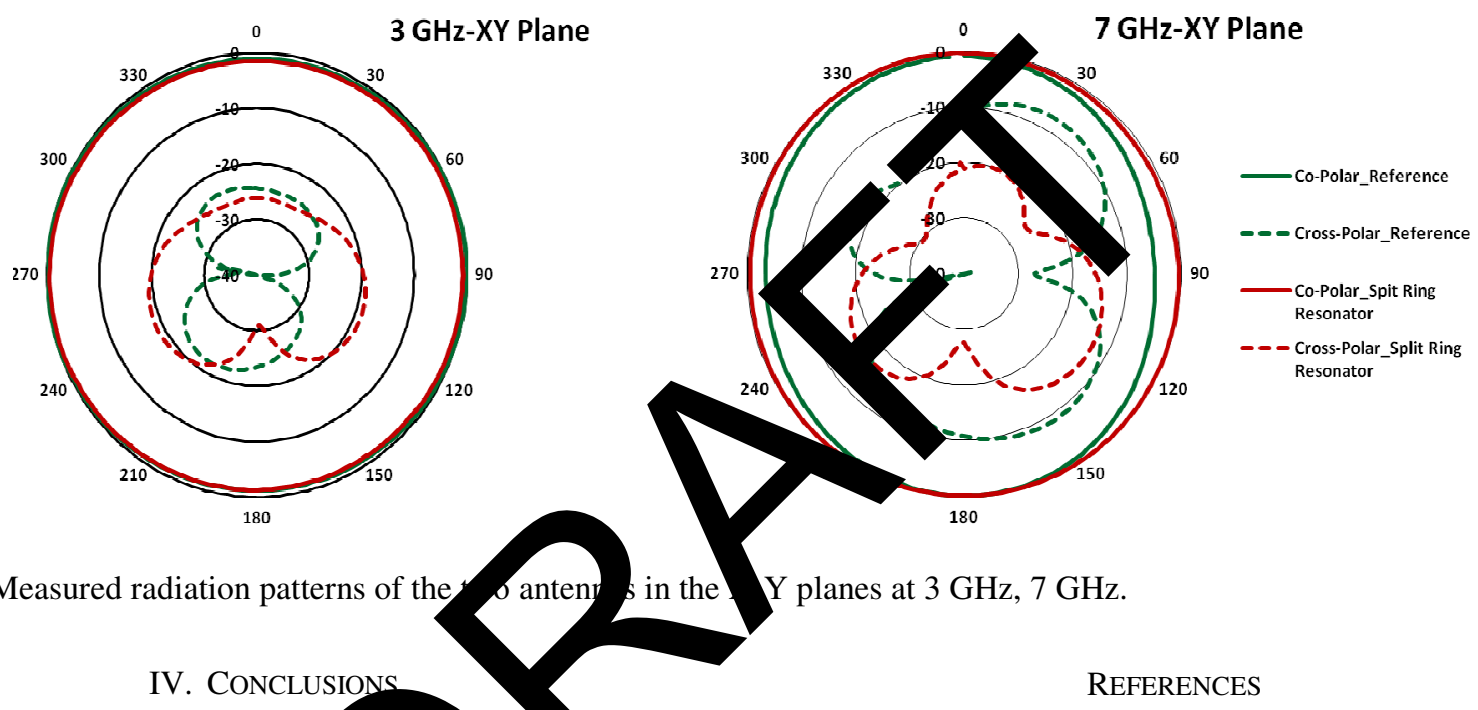

A novel technique is proposed to prove pert ance of polymer based antennas mar of transpa t concuctive materials by introducing split ring sonators in the radiating patch portion of the antenna in this a UW antenna. The results showed that the proposed tec. can be used to increase the gain of the antenna and hus improve the performance of the transparent polymer UWB antenna. The increased gain makes the radiation pattern slightly directional, however, a nearly omni-directional radiation pattern is still maintained thus ensuring good reception and transmission in all directions. The wide bandwidth achieved further solidifies the effectiveness of this technique for gain enhancement in transparent polymer UWB antennas.

The possibility of further increasing the gain was noted in simulation by increasing the dimensions of the split ring resonator. Also possible is the introduction of other similar shapes to increase the gain further but this has been left to future works.

[1] T. Peter and R. Nilavalan, "Study on the performance deterioration of flexible UWB antennas," Antennas and Propagation Conference Loughborough (LAPC'09), pp. 669-672, 2009.

[2] Peter, T.; Sun, Y.Y.; Yuk, T.I.; AbuTarboush, H.F.; Nilavalan, R.; Cheung, S.W.; , "Miniature transparent UWB antenna with tunable notch for green wireless applications," Antenna Technology (iWAT), 2011 International Workshop on , vol., no., pp.259-262, 7-9 March 2011

[3] T. Peter, R. Nilavalan, H. F. AbuTarboush, and S. W. Cheung, "A Novel Technique and Soldering Method to Improve Performance of Transparent Polymer Antennas," IEEE Antennas and Wireless Propagation Letters, vol.9, no., pp.918-921, 2010.

[4] Mias, C.; Tsakonas, C.; Prountzos, N.; Koutsogeorgis, D.C.; Liew, S.C.; Oswald, C.; Ranson, R.; Cranton, W.M.; Thomas, C.B.; ,

"Optically transparent microstrip antennas," Antennas for Automotives (Ref. No. 2000/002), IEE Colloquium on , vol., no., pp.8/1-8/6, 2000.

[5] Katsounaros, A.; Hao, Y.; Collings, N.; Crossland, W.A.; , "Optically transparent antenna for Ultra Wide-Band applications," Antennas and Propagation, 2009. EuCAP 2009. 3rd European Conference on, vol., no., pp.1918-1921, 23-27 March 2009.

[6] J.D. Jackson, "Classical Electrodynamics”, John Wiley \& Sons 1999.

\section{ACKNOWLEDGMENT}

The authors wish to acknowledge Solutia Inc., St. Louis, Missouri, USA for their support in providing the AgHT-8 films used in this research. 